\title{
Hilft Akupressur?
}

\begin{abstract}
Australische Anästhesiologen randomisierten 365 schwangere Frauen in zwei Gruppen. Während der Geburt erhielten sie entweder Akupressur oder eine Scheinbehandlung.

- Die Akupuressur erfolgte bilateral mittels eines Armbands, dass am Punkt P6 (einen Finger breit proximal des Handgelenks) Druck ausübte. Die Scheinbehandlung bestand aus einem Armband, das keinen solchen Druck applizierte. Die Inzidenz von Übelkeit und Erbrechen war in der Verumgruppe $53 \%$ und in der Kontrollgruppe $45 \%$. Alle weiteren Messgrößen wie Antiemetikabedarf zeigten eine ähnliche Tendenz. Die Autoren meinen daher, dass Akupressur bei dieser Indikation nicht wirksam sei.

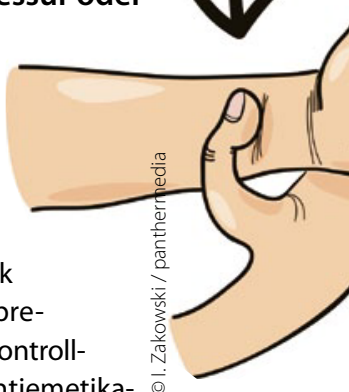
sitive Ergebnisse. Fazit: (Nicht nur) in der Komplementärmedizin löst sich ein vermeintlich positiver Effekt nicht selten in Wohlgefallen auf, wenn man den Bias auf ein Minimum reduziert.
\end{abstract}

E. ERNST -

$$
\text { Kommentar }
$$

Zu diesem Thema gibt es bereits eine ganze Reihe von Studien. Ihre Ergebnisse sind jedoch sehr widersprüchlich, sodass auch ein
- A. Sinha et al.

A randomised, double-blinded, placebo-controlled study of acupressure wristbands for the prevention of nausea and vomiting during labour and delivery. Int. J. Obstet. Anesthesia 20 (2011) 110-117

\section{B-Vitamine schützen nicht vorm Schlaganfall}

Eine Vielzahl von epidemiologischen Studien zeigt, dass erhöhte Homocysteinspiegel im Serum Marker für ein erhöhtes Risiko von Herzinfarkt und Schlaganfall darstellen. Die meisten Beobachtungsstudien hatten nahegelegt, dass eine Substitution von Vitamin B6, B12 und Folsäure zu einer Reduktion von Schlaganfällen und Herzinfarkten führt. Dies konnte allerdings später in großen, randomisierten, placebokontrollierten Studien nicht repliziert werden.

— Die vorliegende Arbeit ist eine kritische Literaturübersicht, welche die randomisierten, doppelblinden, kontrollierten Studien zum Einsatz von BVitaminen zur Prävention vaskulärer Ereignisse und bis dato publizierte $\mathrm{Me}$ taanalysen zusammenfasst .

Es gibt bisher zehn randomisierte, doppelblinde, kontrollierte Studien zum Einsatz von Vitamin B6, B12 oder Folsäure in der Primär- und Sekundärprä- vention vaskulärer Ereignisse inklusive Schlaganfall. Die Studien umfassen zusammen 47460 Patienten. Mit einer Ausnahme waren alle Studien negativ. Lediglich in der HOPE-2-Studie ergab sich eine 24\%ige relative Risikoreduktion für den Endpunkt Schlaganfall. Allerdings wurden in dieser Studie auch Statine eingesetzt, sodass es schwer ist, den isolierten Effekt der Behandlung mit B-Vitaminen und Folsäure zu evaluieren.

Der Autor hat darüber hinaus sechs große Metaanalysen tabellarisch dargestellt, die zwischen acht und 17 prospektive, randomisierte Studien auswerten. In keiner dieser Metaanalysen war die Vitaminsubstitution besser wirksam als eine Behandlung mit Placebo.

Eine Substitution von B-Vitaminen und Folsäure bei Patienten mit erhöhtem Homocystein ist weder in der Primär- noch in der Sekundärprävention in der Lage, vaskuläre Ereignisse und Schlaganfälle zu verhindern.

\section{Kommentar}

Diese Übersicht setzt jetzt hoffentlich den Schlusspunkt unter die seit mehr als zehn Jahren laufende Diskussion zu der Frage, ob die Vitamine B6 und B12 sowie Folsäure in der Lage sind, bei Patienten mit erhöhten Homocysteinspiegeln die Rate vaskulärer Ereignisse zu vermindern. Die Ergebnisse zeigen eindeutig, dass erhöhtes Homocystein zwar ein Biomarker ist, aber kein kausaler Zusammenhang zwischen erhöhten Homocysteinspiegeln und der Prävalenz von Schlaganfällen und Herzinfarkten besteht. Dieser Zusammenhang gilt sowohl für die Primär- als auch für die Sekundärprävention.

H.-C. DIENER =

\section{- G. Saposnik}

The role of vitamin B in stroke prevention: a journey from observational studies to clinical trials and critique of the VITAmins TO Prevent Stroke (VITATOPS). Stroke 42 (2011) 838-842 